\title{
COMPARACIÓN ENTRE LOS SCORES DE RIPASA Y ALVARADO MODIFICADO EN EL DIAGNÓSTICO DE APENDICITIS AGUDA, 2015-2016.
}

\section{COMPARISON BETWEEN THE SCORES OF RIPASA AND ALVARADO MODIFIED IN THE DIAGNOSIS OF ACUTE APPENDICITIS, 2015-2016}

\section{ARTíCULO ORIGINAL}

\author{
Milenka Conde-Quintana ${ }^{1, a}$, Irvin Garcia-Donayre ${ }^{\text {a, }}$ Gerardo Parvina-Quezada ${ }^{\text {,a }}$. \\ 1. Dirección Regional de Salud Ayacucho. Ayacucho, Perú. \\ a. Médico cirujano.
}

\section{Correspondencia:}

Milenka Conde-Quintana

Dirección: Urb. Villa del Médico B-7

Ica, Perú.

Teléfono: (51)956747941

Correo Electrónico:

milenka_live_1@hotmail.com

\section{Contribuciones De Autoría:}

MAII, GAAD, LMGB, CMB

participaron en el diseño del estudio, el análisis de los datos, revisaron críticamente el artículo y aprobaron la versión final

Conflicto De Intereses: No declarados.

\section{Financiamiento: Autofinanciado}

Como Citar

Conde-Quintana M, García-Donayre Irvin,Parvina-Quezada G. Comparación entre los scores de Ripasa y Alvarado modificado en el diagnóstico de apendicitis aguda, 2015-2016. 2016. Rev méd panacea. 2017; 6 (2): $69-72$

Recibido: 10-05-2017

Aceptado: 15-06-2017

Publicado: 20-06-2017

\section{RESUMEN:}

Objetivo: Determinar que score tiene mayor sensibilidad y especificidad entre el de RIPASA y el de ALVARADO modificado para corroborar el diagnóstico de Apendicitis Aguda en pacientes de 16 a 60 años de edad, Hospital Regional de Ica del 1 de octubre del 2015 al 30 de setiembre del 2016. Material y Metodos: Se realizó un estudio descriptivo, transversal y retrospectivo de 150 historias, se apoyo con los programas estadístico SPSS V 22.0; Microsoft Word 2013 y Microsoft Excel. Resultados: La sensibilidad, especificidad, VPP y VPN del score de RIPASA fue $88,7 \%, 77,8 \%, 98,4 \%$ y $30,4 \%$ respectivamente (para un score $\geq 7,5$ ) y para el score de Alvarado fue $71,6 \%, 77,8 \%, 98,1 \%$ y $14,9 \%$ respectivamente (para un score $\geq 7$ ), El área bajo la curva ROC del score de RIPASA fue de 0.848 , superior a la de Alvarado de 0.81 .

Conclusiones: La efectividad diagnóstica del score de RIPASA fue $84.8 \%$ y del score de Alvarado Modificado de $81 \%$, demostrando mayor exactitud como prueba diagnóstica.

Palabras Claves: Apendicitis aguda, score Alvarado modificada, score de RIPASA. (fuente: DeCS BIREME).

\section{ABSTRACT:}

Objetive: To determine which score has greater sensitivity and specificity between RIPASA and modified ALVARADO to corroborate the diagnosis of acute appendicitis in patients 16 to 60 years of age, Regional Hospital of Ica from October 1, 2015 to September 30 2016. Material And Methods: We carried out a descriptive, transversal and retrospective study of 150 histories, supported with the statistical programs SPSS $\checkmark$ 22.0; Results: The sensitivity, specificity, VPP and NPV of the RIPASA score were $88.7 \%, 77.8 \%, 98.4 \%$ and $30.4 \%$, respectively (for a score $\geq 7.5$ ) And the Alvarado score was $71.6 \%, 77.8 \%, 98.1 \%$ and $14.9 \%$, respectively (for a score 27 ). The area under the ROC curve of the RIPASA score was 0.848 , higher To that of Alvarado of 0.81 .

Conclusions: The diagnostic effectiveness of the RIPASA score was $84.8 \%$ and the modified Alvarado score was $81 \%$, demonstrating greater accuracy as a diagnostic test.

Keywords: Acute appendicitis, modified Alvarado score, RIPASA score. (source: MeSH NLM). 


\section{INTRODUCCIÓN}

La apendicitis aguda es el cuadro quirúrgico más frecuente en tópico de emergencia de cirugía general del Hospital Regional de Ica, y en general en todas las instituciones de salud a nivel mundial. Su importancia radica, por tanto, en la elevada incidencia y morbilidad postquirúrgica que engloba (1). El $25 \%$ de los pacientes que acuden a emergencia con abdomen agudo quirúrgico son diagnosticados de apendicitis aguda, desarrollándose diferentes estudios para un diagnóstico precoz y oportuno $(2,3)$. El riesgo de la población de padecer apendicitis aguda es de 8,6\% para los hombres y $6,7 \%$ para mujeres, siendo más frecuente entre los 10 y los 30 años de edad. Cuando no se sospecha ni se diagnostica correctamente, esta enfermedad puede evolucionar hacia la perforación; esta complicación puede variar entre el 15 y el $37 \% .(4,5)$.

Con la finalidad de disminuir la morbilidad e incluso la mortalidad asociada a la apendicitis aguda, a través de los años han surgido diferentes maneras de valorar esta enfermedad y poder predecir que pacientes deben ser sometidos a cirugía, los propuestos han sido algunos sistemas de scores como el de Alvarado, de Omhan, de Fenyo - Lindberg, el recuento de leucocitos, la ultrasonografía (US), la tomografía axial computarizada (TAC), la resonancia magnética, algunos modelos combinando criterios clínicos, bioquímicos y de imágenes; como la de Tzanakis, la de RIPASA (Hospital Raja Isteri Pengiran Anak Saleha-norte de Borneo, Asia); cada uno de ellos con una sensibilidad y especificidad diferente, siendo las más altas con la TAC, sin embargo muy costosas y no accesibles en la gran mayoría de hospitales. Varios estudios han mostrado que los scores brindan al cirujano una información valiosa en su toma de decisión quirúrgica.(3)

La escala de Alvarado modificada permite que los pacientes que consultan al Servicio de Urgencias con dolor abdominal en la fosa iliaca derecha puedan clasificarse en 3 grupos, de acuerdo con la probabilidad de tener apendicitis, en el 2010, en el Hospital RIPAS, al norte de Borneo, en Asia, elaboraron una escala mejorada para diagnóstico precoz de apendicitis aguda, denominándola score RIPASA, con mejor sensibilidad y especificidad $(6,7,8)$.

Consideramos que la aplicación de estos scores en los servicios de Cirugía en emergencia por parte de los médicos puede ofrecer una ventaja para la toma de decisiones con respecto a operar o no al paciente con diagnóstico de apendicitis aguda, por ello determinamos nuestro objetivo: Identificar que score tiene mayor sensibilidad y especificidad entre el de RIPASA y el de ALVARADO modificado para corroborar el diagnóstico de Apendicitis Aguda en pacientes de 16 a 60 años de edad, que fueron intervenidos quirúr-gicamente de emergencia, en el Hospital Regional de Ica durante el 1 de octubre del 2015 al 30 de setiembre del 2016; además comparar el valor predictivo positivo y negativo del score de RIPASA y del score de ALVARADO modificado para corroborar el diagnóstico de Apendicitis Aguda.

\section{MATERIAL Y MÉTODOS}

Se realizó un estudio descriptivo, transversal y retrospectivo de 340 historias clínicas. Se empleó el método de recolección de datos para lo cual se elaboró una ficha en donde se registraron datos como: Sexo, edad, tiempo de enfermedad, el score modificado de Alvarado, score de RIPASA, diagnóstico pre-operatorio, y diagnóstico postope-ratorio.
Se utilizó los programas estadístico SPSS V 22.0; Microsoft Word 2013 y Microsoft Excel. Se elaboraron cuadros y gráficas. Se calculó la Sensibilidad, Especificidad, Valor predictivo positivo (VPP), Valor predictivo negativo (VPN), Medidas de tendencia central, de dispersión y se confeccionó tablas de frecuencias.

La muestra de 150 Historias clínicas se obtuvo por muestreo aleatorio simple, para una población total de 340 pacientes.

$$
\begin{aligned}
& n=\frac{N z^{2} p q}{(N-1) e^{2}+z^{2} p q} \\
& n=\frac{(340)\left(1.96^{2}(0.5)(0.5)\right.}{(340-1)\left(0.06^{2}\right)+\left(1.96^{2}\right)(0.5)(0.5)}
\end{aligned}
$$

$n=149.73$

\section{RESULTADOS}

Se observó que el $59.3 \%$ fueron de sexo masculino y $40.7 \%$ sexo femenino, la relación hombre/mujer fue 1.46 (Figura 1).

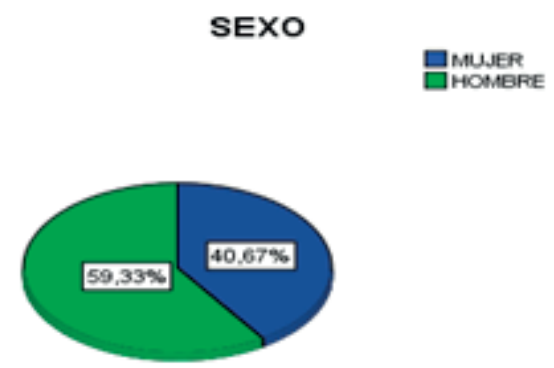

Al aplicar la regla de sturges $(k=1+3.32 \log 150)$ para intervalos de edad (Tabla 1 ) se encontró que el $56 \%$ correspondió a edades entre 16 y 29 años.

El principal motivo de consulta fue dolor en fosa iliaca derecha en un $100 \%$, discriminando dolor de inicio en epigastrio (dolor migratorio-cronología de Murphy) en un 75,3\%.

Se observó que los scores promedios obtenidos a la admisión, aumentaban de manera progresiva a medida que avanza el estadio de la apendicitis.

\begin{tabular}{|c|c|c|c|}
\hline Intervalo & Frecuencia & Porcentaje & PA \\
\hline$\left[\begin{array}{ll}16 & 22\end{array}\right]$ & 48 & 32.0 & 32.0 \\
\hline$\left[\begin{array}{ll}23 & 29\end{array}\right]$ & 36 & 24.0 & 56.0 \\
\hline$\left[\begin{array}{ll}30 & 36\end{array}\right]$ & 29 & 19.3 & 75.3 \\
\hline$\left[\begin{array}{ll}37 & 43\end{array}\right]$ & 18 & 12.0 & 87.3 \\
\hline$\left[\begin{array}{ll}44 & 50\end{array}\right]$ & 12 & 8.0 & 95.3 \\
\hline$\left[\begin{array}{ll}51 & 57\end{array}\right]$ & 7 & 4.7 & 100. \\
\hline$\geq 58$ & 0 & 0.0 & 100. \\
\hline Total & 150 & 100 & \\
\hline
\end{tabular}

Tabla 1. Distribución por Edad de pacientes con diagnóstico de AA.

PA, porcentaje acumulado 
(Tabla. 2) lo cual estaría relacionada al mayor compromiso inflamatorio, que aumentaría la aparición progresiva de su sintomatología, Al asociar RIPASA

dicotomizado (con punto de corte 7.5) con cada estadio de apendicitis aguda se obtuvo una sensibilidad y especificidad respectivamente en AA CONGESTIVA de $85,7 \%$ y 15,4. En AA FLEMONOSA de $81.3 \%$ y $13,8 \%$. En AA NECROSADA de $92.7 \%$ y $20 \%$. Asociando ALVARADO modi-ficado dicotomizado (con el punto de corte 7) con cada estadio de apendicitis aguda se obtuvo una sensibilidad y especificidad respectivamente en: AA CONGE-STIVA de $85,7 \%$ y $32,2 \%$. En AA FLEMONOSA de $60.4 \%$ y $27.5 \%$. En AA NECROSADA de $76.4 \%$ y $35.8 \%$.

\begin{tabular}{|c|c|c|c|c|c|c|}
\hline \multicolumn{7}{|c|}{ de apendicitis aguda según estadio. } \\
\hline & AA & & AA & & AA & \\
\hline & Edematosa & & Flemonosa & & Necrosada & \\
\hline & 5 & $\mathrm{E}$ & 5 & E & 5 & $E$ \\
\hline RIPASA & 85.7 & 15.4 & 81.3 & 13.7 & 92.7 & 20 \\
\hline \multicolumn{7}{|l|}{$\geq 7.5$} \\
\hline & & & & & & \\
\hline ALVARADO & 85.7 & 32.2 & 60.4 & 27.5 & 76.4 & 35.8 \\
\hline$\geq 7$ & & & & & & \\
\hline
\end{tabular}

S, sensibilidad (\%); E, especificidad (\%).

El score de RIPASA para corroborar el diagnóstico final de apendicitis aguda tuvo una sensibilidad de $88,7 \%$, especificidad $77,8 \%$, VPP $98,4 \%$, VPN 30,4\%, compatible con apendicitis aguda para un score $\geq 7,5$ (Tabla 3 ).

\begin{tabular}{|l|c|c|c|c|}
\hline \multicolumn{4}{|c|}{ Tabla 3. Poder predictivo del score de RIPASA y Alvarado modificado } \\
\cline { 3 - 5 } & S & E & VPP & VPN \\
\hline $\begin{array}{l}\text { RIPASA } \\
\geq 7,5\end{array}$ & 88,7 & 77,8 & 98,4 & 30,4 \\
\hline $\begin{array}{l}\text { ALVARADO } \\
\geq 7\end{array}$ & 71,6 & $77,8 \%$ & 98,1 & 14,9 \\
\hline
\end{tabular}

$\mathrm{S}=$ sensibilidad (\%); E=especificidad (\%), VPP=valor predictivo positivo (\%), VPN=valor predictivo negativo (\%).

El score de Alvarado Modificado para corroborar el diagnóstico final de apendicitis aguda tuvo una sensibilidad de $71,6 \%$, especificidad $71,6 \%$, VPP $77,8 \%$ y VPN de, $98,1 \%$, $14,9 \%$, respectivamente, compatible con apendicitis aguda para un score $\geq 7$.

\section{DISCUSIÓN}

El objetivo principal del proceso de toma de decisiones clínicas es llegar a un diagnóstico preciso en el menor tiempo y costo posible. Con base en la historia y examen clínico, el cirujano debe tomar una decisión muy importante porque una intervención quirúrgica por apendicitis aguda conlleva un riesgo definitivo de mortalidad y morbilidad. Los variados signos y síntomas pueden confundir a los jóvenes cirujanos, quienes son los responsables de hacer el diagnóstico en la emergencia, y pueden llegar a errar en $50 \%$ de las veces $(9,10,11)$
En nuestro estudio la edad más frecuente fue 19 años, el promedio 29.59 años, la desviación estándar de 10.31, la menor y mayor edad fue 17 y 57 respectivamente.

En relación al rendimiento diag-nóstico de los scores estudiados se obtuvo una sensibilidad de $88.7 \%$, para un score $\geq 7.5$, el cual es compatible con apendicitis aguda y una especificidad de $77,8 \%$, para el score de RIPASA. Un valor predictivo positivo de $98,4 \%$, es decir que pacientes que tengan prueba positiva, en un $98,4 \%$ realmente tienen Diagnóstico de Apendicitis aguda. $Y$ un valor predictivo negativo de $30.4 \%$, significando que la probabilidad que un sujeto con score de RIPASA negativo, no tenga realmente Apendicitis aguda en un $30.4 \%$.

Para el score de Alvarado modificado se obtiene una sensibilidad de $71.6 \%$, para un score $\geq 7$, el cual es compatible con apendicitis aguda y una especificidad de $77,8 \%$. Así mismo pacientes con score de Alvarado modificado positiva, realmente tenga el diagnóstico de apendicitis aguda en un $98,1 \%(\mathrm{VPP})$, y un $14.9 \%(\mathrm{VPN})$, que con score Alvarado modificado negativo, una persona realmente no tenga diagnóstico de apendicitis aguda.

Del Cóndor I (3), en el Hospital Regional Docente de Trujillo. TrujilloPerú, en el estudio de Comparación entre los Scores de RIPASA y Alvarado en el diagnóstico de Apendicitis Aguda encontró que en 352 pacientes la sensibilidad, especificidad, VPP y VPN del score de RIPASA fue $99,70 \%, 42,86 \%$, 96,49\% y 90\% respectivamente (para un score $\geq 7,5)$ y para el score de Alvarado fue $91,84 \%, 42,86 \%, 96,20 \%$ y $25 \%$ res-pectivamente (para un score $\geq 7$ ). Resultados similares encontraron Ron A et al (12) y Ospina J, Barrera L, Manrrique F (13), con respecto al score de Alvarado.

Reyes $\mathrm{N}$ et al (5), en el Hospital General de México en un estudio sobre Precisión diagnóstica de la escala RIPASA para el diagnóstico de apendicitis aguda: Análisis Comparativo con la escala de Alvarado modificada encontró que en 70 paciente el score RIPASA presentó una sensibilidad de $91,2 \%$ y especificidad de $84,6 \%$ y el score de Alvarado modificado presentó una sensibilidad de $89,5 \%$ y especificidad de 69,2\%. Resultados similares encontraron Ricci L, Ferreyra C, Cordoba M, Rios A, Stasti M (14) y Orbia M (15), en lo que concierne a la escala de Alvarado.

Alnjadat I, et al (9) en el Hospital Princesa Haya, Aqaba, Jordania en un estudio sobre Puntuación Alvarado vs RIPASA en el diagnóstico de apendicitis aguda encontró que en 600 pacientes encontraron una sensibilidad, especificidad VPP y VPN para los scores de RIPASA y Alvarado de $93,2 \%$ vs $73,7 \%, 61,8 \%$ vs $68,6 \%, 92,2 \%$ vs $92 \%$ y $64,9 \%$ vs $34,8 \%$ respectivamente. Estos resultados son concordantes también con los de Alarcón N (16), en lo que se refiere a la escala de Alvarado.

Todos ellos muestran que el rendimiento obtenido en nuestra serie se encuentra entre los valores reportados por otras series, lo cual demuestra de que es posible evaluar pacientes en una manera racional, usando un simple score diagnóstico que podría indicar cuales pacientes deberían ser observados y cuáles deberían tener cirugía, además se muestra que este nuevo score de RIPASA tuvo mejor rendimiento en comparación con el ya conocido y extensamente usado score de Alvarado.

Finalmente al comparar los scores RIPASA y Alvarado se nota superioridad con respecto al score de RIPASA sobre el score de Alvarado modificado 


\section{BIBLIOGRAFÍA}

1. Trujillo Y, Contreras C: Score diagnóstico modificado en el diagnóstico de Apendicitis Aguda-revista médica carriónica[Revista on-line] 2015[Consultado 17 mayo 2015]; 2(1). Disponible en: http://cuerpomedico.hdosdemayo.gob.pe/index.php/revistamedicacarrionica/article/view/41

2. Andrade M., Astudillo J, Bermeo J. Comparación de la eficacia diagnostica para apendicitis de la escala de Alvarado versus ecografía teniendo como referencia el resultado histopatológico en pacientes mayores de 12 años ingresados al servicio de emergencia del hospital Vicente Corral Moscoso desde enero del 2011 hasta diciembre 2011, Cuenca - Ecuador 2012.[Tesis de Doctoral]. Cuenca: Escuela de posgrado, Universidad de Cuenca; 2013.

3. Del Cóndor I. Comparación entre los scores de Ripasa y Alvarado en el diagnóstico de apendicitis aguda en pacientes de 14 a 60 años de edad en el Hospital Regional Docente de Trujillo. [Tesis de Doctoral]. Trujillo: Escuela de posgrado, Universidad Privada Antenor Orrego; 2014

4. Chong C, et al. Comparison of Ripasa and Alvarado scores for the diagnosis of acute appendicitis 2011;52(5):340-345

5. Reyes N, Zaldívar F, Cruz R, Sandoval M, Gutiérrez C, Athié C: Precisión diagnóstica de la escala RIPASA para el diagnóstico de apendicitis aguda: análisis comparativo con la escala de Alvarado modificada-revista medigraphic [Revista on-line] 2012 [Consultado 17 mayo 2015]; 34(2). Disponible en:

http://www.medigraphic.com/pdfs/cirgen/cg-2012/cg122b.pdf

6. Zavala L. Score diagnóstico de la apendicitis aguda. Evaluación de la escala de Alvarado. Estudio prospectivo realizado en el hospital del niño "Francisco de Ycaza Bustamante" 2012-2013. [Tesis de Doctoral]. Guayaquil: Escuela de posgrado, Universidad Católica de Santiago de Guayaquil; 2014.

7. Oronoz L. Evaluación de la escala de Alvarado en pacientes con dolor abdominal sugestivo de apendicitis aguda. [Tesis de Doctoral]. Naguanagua: Direcciòn de posgrado, Universidad de Carabobo; 2011

8. Klabtawee W, Saensak W, Khetsoongnern A, Piriyasupong T. Accuracy of RIPASA and Modified RIPASA score Comparing with Alvarado score for Diagnosis of Acute Appendicitis and Complication of Acute Appendicitis. KhonKaen Medical Journal 2011;35(1):40-50
9. Alnjadat I, Abdallah B. Alvarado versus RIPASA score in diagnosing acute appendicitis. Rawal Medical Journal 2013;38(2):147-151.

10. Hernández L, Domínguez D. Sensibilidad y especificidad de la escala de Alvarado en apendicitis aguda en el Hospital Regional de Alta Especialidad de Veracruz- revista medigraphic [Revista on-line] 2012 [Consultado 17 mayo 2015]; 34(3). Disponible en:

http://www.medigraphic.com/pdfs/ cirgen/cg-2012/cg123e.pdf 11. Cedillo E, Santana I, González R, Onofre J, Gartz G. Sensibilidad y especificidad de la escala de Alvarado en el diagnóstico de apendicitis aguda comparada con TAC o ultrasonido en las primeras 24 horas de evolución- revista medigraphic [Revista on-line] 2012 [Consultado 17 mayo 2015]; 34(2). Disponible en:http://www.medigraphic.com/pdfs/cirgen/cg-2012/cg122c.pdf

12. Ron A, Saleme E, Guerrero T, Hernández, Montiel H, Olvera C. Utilidad de la escala de Alvarado para el estudio del dolor abdominal inespecífico en el Departamento de Urgencias del Centro Médico ABC-revista medigraphic [Revista on-line] 2013 [Consultado 17 mayo 2015]; 58(2). Disponible en: http://www.medigraphic.com/pdfs/abc/bc-2013/bc132c.pdf

13. Ospina J, Barrera L, Manrique F. Utilidad de una escala diagnóstica en casos de apendicitis aguda- Rev Colomb Cir.[Revista on-line] 2011[Consultado 17 mayo 2015]; 26. Disponible en:

http://www.scielo.org.co/pdf/rcci/v26n4/v26n4a2.pdf

14. Ricci L, Ferreyra C, Córdoba M, Rios A, Statti M. Apendicitis aguda según los criterios de Alvarado- Rev Argent Cirug.[Revista on-line] 2015[Consultado 17 mayo 2015]; 107(2). Disponible en: http://www.aac.org.ar/revista/2015/107/Junio/3.pdf

15. Orbea V. Utilidad de la escala de Alvarado en el diagnóstico temprano de apendicitis aguda; Hospital provincial Puyo Abril Diciembre 2009. [Tesis de Doctoral]. Riobamba: Escuela de posgrado, Escuela Superior Politécnica de Chimborazo; 2010.

16. Alarcón N: Asociación entre Escala de Alvarado y diagnóstico de apendicitis aguda complicada y no complicada según anatomía patológica en el Centro Médico Naval.-revista horizonte médico[Revista on-line] 2012 [Consultado 17 mayo 2015]; 12(2). Disponible en: http://www.horizontemedicina.usmp.edu.pe/index.php/horizontemed/article/view/99

AGRADECIMIENTOS: Al Dr. Anselmo Magallanes Carrillo, por su ayuda desinteresada y al personal que labora en el Hospital Regional de Ica. 